\title{
Separating the scattering and absorption coefficients using the real and imaginary parts of the refractive index with low-coherence interferometry
}

\author{
Francisco E. Robles and Adam Wax* \\ Department of Biomedical Engineering and Medical Physics Program, Duke University, Durham, North Carolina 27708, USA \\ *Corresponding author: a.wax@duke.edu
}

Received April 20, 2010; revised June 28, 2010; accepted July 19, 2010;

posted August 3, 2010 (Doc. ID 127317); published August 17, 2010

\begin{abstract}
We present an analytical method that yields the real and imaginary parts of the refractive index (RI) from lowcoherence interferometry measurements, leading to the separation of the scattering and absorption coefficients of turbid samples. The imaginary RI is measured using time-frequency analysis, with the real part obtained by analyzing the nonlinear phase induced by a sample. A derivation relating the real part of the RI to the nonlinear phase term of the signal is presented, along with measurements from scattering and nonscattering samples that exhibit absorption due to hemoglobin. (c) 2010 Optical Society of America

OCIS codes: $120.3180,260.2030,300.1030,110.4500$.
\end{abstract}

Substantial efforts have been made to quantitatively measure absorption of biological chromophores in vivo for diagnosis [1-ㅜㄹ. Unfortunately, several drawbacks have been encountered primarily dealing with the limitation of the number of physical parameters that may be independently measured and determined using analytical models. For example, in spectroscopic optical coherence tomography (SOCT), some promising results for quantifying absorption in vivo have been reported [2-4] , but these have been limited because SOCT measures the total attenuation coefficient, and the absorption and scattering contributions have not been separated without $a$ priori information. Consequently, in order to assess absorption, models must include additional parameters, such as packing factors and anisotropic coefficients, which results in low confidence intervals, thereby hindering quantification [5]. Rather than using attenuation, some researchers have used phase information as a means of obtaining concentrations of chromophores [6]. These methods, however, are often confined to thin, transparent samples and thus are not well suited for in vivo measurements.

In this Letter, we analyze the dispersion of turbid samples using low-coherence interferometric (LCI) signals, in combination with time-frequency (TF) analysis, to retrieve the real and imaginary parts of the RI and quantify absorption independently of scattering contributions and without a priori information. Using a supercontinuum broadband light source, we demonstrate that this method yields wideband absorption spectral profiles with high spectral resolution, in addition to RI profiles. By isolating the contribution due to absorption, the scattering coefficient is also obtained.

To understand how the real and imaginary parts of the RI may be obtained, consider a Michelson interferometer with a reference field described as $E_{r}(\omega)=S(\omega)$ $\exp \left[i\left(\omega / c_{0}\right) 2 z_{r}\right]$, and a sample field returned by $m$ scatterers written as

$$
E_{s}(\omega)=\sum_{m} \sqrt{I_{s}^{(m)}(\omega)} \cdot e^{i\left(\omega / c_{0}\right) 2 z_{d}} e^{i\left(\omega / c_{0}\right) n(\omega) 2\left(z_{s}^{(m)}-z_{d}\right)},
$$

where $z_{r}, z_{d}$, and $z_{s}$ are the distances from the beam splitter to the reference mirror, dispersive medium, and scatterer, respectively; $S(\omega)$ is the spectrum of the source field; $c_{0}$ is the speed of light in vacuum; and $n(\omega)$ is the real part of the RI of the sample [7]. For simplicity, we assume that there is a single scatterer $(m=1)$, and that the sample can be described by bulk absorption and scattering coefficients $\mu_{a}$ and $\mu_{s}$, respectively; such that the sample field intensity may be written as $I_{s}(\omega)=|S(\omega)|^{2} \exp \left[-\left(\mu_{a}+\mu_{s}\right) 2\left(z_{s}-z_{d}\right)\right]$. Thus, the interferometric signal, after elimination of dc background terms, is given by

$$
\begin{aligned}
\tilde{I}(\omega) & =2 \sqrt{I_{s}(\omega) I_{r}(\omega)} \cdot e^{i\left(\omega / c_{0}\right) 2\left(z^{\prime}-d n(\omega)\right)} \\
& =2 I_{r}(\omega) e^{-\mu_{\mathrm{tot}}(\omega) d} \cdot e^{i\left(\omega / c_{0}\right) 2\left(z^{\prime}-d n(\omega)\right)},
\end{aligned}
$$

where $\quad I_{r}=|S|^{2}, \quad \mu_{\mathrm{tot}}=\mu_{a}+\mu_{s}, \quad z^{\prime}=z_{r}-z_{d}, \quad$ and $d=z_{s}-z_{d}$. Then, to analyze the information contained in the dispersion of the signal, we consider a Taylor series expansion of $n(\omega), n(\omega)=n\left(\omega_{0}\right)+\Delta n(\omega)$, where $n\left(\omega_{0}\right)$ may be evaluated at an arbitrary frequency or wavelength, and $\Delta n$ incorporates all the terms dependent on $\omega$ from the Taylor series expansion. Thus, Eq. (2) may be rewritten as

$$
\begin{aligned}
\tilde{I}(\omega)= & 2 I_{r}(\omega) e^{-\mu_{\mathrm{tot}}(\omega) d} \cdot e^{i\left(\omega / c_{0}\right) \cdot 2\left(z^{\prime}-d n\left(\omega_{0}\right)\right)} \\
& \cdot e^{-i\left(\omega / c_{0}\right) \cdot 2 d \Delta n(\omega)} .
\end{aligned}
$$

As Eq. (3) describes, the measured signal contains three parts: The first part modulates the intensity, which yields spectroscopic information and, hence, access to $\mu_{\text {tot }}$. The second part linearly modulates the phase of the interference signal, which can be processed to yield depth-resolved information, as is commonly done in spectral-domain optical coherence tomography (OCT). Lastly, the third term shows a nonlinear modulation of the phase, which describes dispersion due to the sample. This term is associated with a degradation of the axial resolution in OCT, but it also contains information regarding the wavelength-dependent changes in the real 
part of the RI. Previous efforts to quantify absorption [2-4] and scattering [8] have used only the intensity and the linear phase terms and have ignored the information contained in the dispersive term.

Algorithms have been developed for removing dispersion inherent in an optical system or due to a sample. One such algorithm, presented by Zhu et al. [9], fits the unwrapped phase of the signal to a line of the form $\phi=\left(\omega / c_{0}\right) L-2 \pi m$, where $m$ is a positive integer and $L$ is the best estimate of the constant $2\left(z^{\prime}-d n_{0}\right)$ in Eq. (3). The residual phase, $\Delta \phi$, which is the difference between the unwrapped phase and $\phi$, is then subtracted from the phase of subsequent acquisitions to obtain dispersion-free signals. Therefore, the residual phase is related to the real part of the RI by $\Delta \phi=\left(\omega / c_{0}\right) 2 d \Delta n(\omega)$. Then, by principles of causality, and by using KramersKroning (KK) relations, the real part of the RI may be expressed in terms of the imaginary part, which is proportional to the absorption coefficient. Note that $\Delta n$ and $\mu_{a}$ are quantities linearly dependent on concentration.

Let us consider the quantification of hemoglobin $(\mathrm{Hb})$ concentration for two cases. In the case where the scattering coefficient is negligible (case A), $\mu_{\text {tot }}=\mu_{a}=2 \kappa \omega / c_{0}$, and the imaginary part of the RI $(\kappa)$ can be directly obtained using $\mathrm{TF}$ analysis, while the real part of the RI can be calculated from $\Delta \phi$. Both independent measurements may be used to measure the concentration of chromophores. This case, however, is highly idealized and only serves as a base of comparison for a more biologically relevant example, given by case $\mathrm{B}$, where $\mu_{s}$ is not negligible and is unknown. For this case (B), the measured attenuation $\left(\mu_{\text {tot }}\right)$, obtained from TF analysis, will overestimate chromophore concentrations. Erroneous estimations may be avoided by introducing other free parameters, but this process yields results with low confidence intervals [5]. On the other hand, for case $\mathrm{B}, \Delta \phi$ will give the same results as in the idealized case A, for equal chromophore concentrations, regardless of the influence of scattering. Thus, because the real RI of the absorber is calculated from $\Delta \phi$, the imaginary part of the RI, which is proportional to $\mu_{a}$, may be determined independently of scattering using the KK relation. Lastly, by using the determined $\mu_{a}$ and the measured $\mu_{\text {tot }}$ for case B, the scattering coefficient may also be obtained, simply by subtraction, $\mu_{s}=\mu_{\text {tot }}-\mu_{a}$.

To demonstrate the validity of this approach, LCI signals from scattering and nonscattering $\mathrm{Hb}$ phantoms were analyzed. The system used a typical Michelson interferometer geometry, where broadband light from a supercontinuum source (Fianium, Eugene, Oregon), is focused onto the sample. A series of filters is used to shape the source spectrum, thus delivering a total power of $\sim 1.5 \mathrm{~mW}$, with a spectral range from $\sim 450$ to $700 \mathrm{~nm}$, onto the sample (see inset of Fig. 2). Scattered light returned from the sample is mixed with a reference field and relayed to a spectrometer for detection. With the center wavelength $\lambda_{0}=575 \mathrm{~nm}$, spectral resolution $\delta \lambda=$ $0.2 \mathrm{~nm}$, and bandwidth $\Delta \lambda=240 \mathrm{~nm}$, we operate in a spectral region where the light experiences high degrees of absorption from $\mathrm{Hb}$, which, in turn, results in significant changes in the real part of the RI.

First, the absorption due to the sample is analyzed to obtain the imaginary part of the RI. For case A, fully
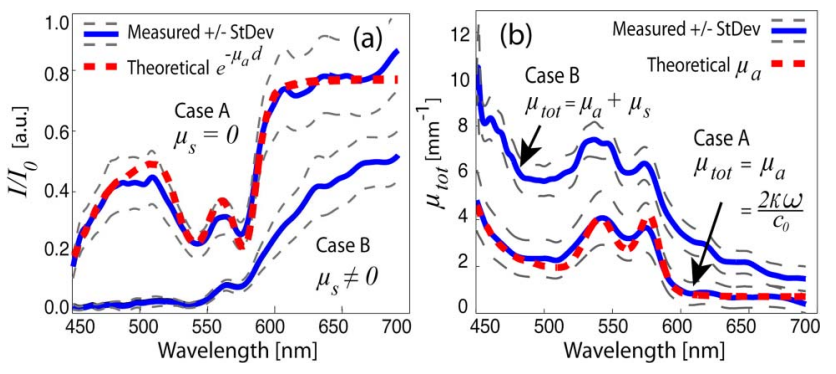

Fig. 1. (Color online) (a) Measured cumulative absorption and (b) total attenuation coefficient of the $\mathrm{Hb}$ phantoms without scattering (case A) and with scattering (case B). The theoretical absorption with (a) $d=400 \mu \mathrm{m}$ and $C=40 \mathrm{~g} / \mathrm{L}$ and (b) corresponding absorption coefficient are also plotted.

oxygenated Hb (Sigma-Aldrich, St. Louis, Missouri), diluted to $40 \mathrm{~g} / \mathrm{L}$ in water, was used. For case $\mathrm{B}, 10.75 \%$ by volume of the aqueous solution in the phantom was made up of $10 \%$ intralipid (IL), which possesses a scattering coefficient 4 orders of magnitude greater than its absorption coefficient. The solutions were then placed between microscope slides with spacers used to set the sample thickness to $400 \mu \mathrm{m}$. For comparison, the total $\mathrm{Hb}$ absorption from these samples is approximately equivalent to a $100-\mu$ m-thick sample of whole blood $(150 \mathrm{~g} / \mathrm{L} \mathrm{Hb})$ or a few millimeters of tissue $(\sim 2-7 \mathrm{~g} / \mathrm{L})$. Depth-resolved spectral profiles were acquired from the interferometric data using the dual window method, as described in [4]. To obtain concentration values, individual $\mathrm{Hb}$ spectra were acquired and normalized by a reference spectrum obtained from the average of 10 acquisitions of a pure water sample. The logarithm of the ratio was taken, yielding, $\ln \left(I / I_{0}\right)=-\mu_{a} d=-C d \varepsilon(\omega) / \mathrm{MW}$, where $\varepsilon(\omega)$ is the molar extinction coefficient, which is independent of $\mathrm{Hb}$ concentration $(C), I_{0}$ is the reference water spectrum, and $\mathrm{MW}$ is the molecular weight of $\mathrm{Hb}(\mathrm{MW}=$ $64,500 \mathrm{~g} / \mathrm{mol}$ ). Because $\varepsilon(\omega)$ is known [10], a linear least-squares method was used to estimate $C$. The average of ten measurements for case A gave $C=40.48+/$ $2.30 \mathrm{~g} / \mathrm{L}$, in good agreement with the known concentration. For case B, $C=58.13+/-4.35 \mathrm{~g} / \mathrm{L}$, which, as expected, overestimates the actual $\mathrm{Hb}$ concentration due to the contribution to attenuation from scattering, which is not accounted for in this simple model. The measured
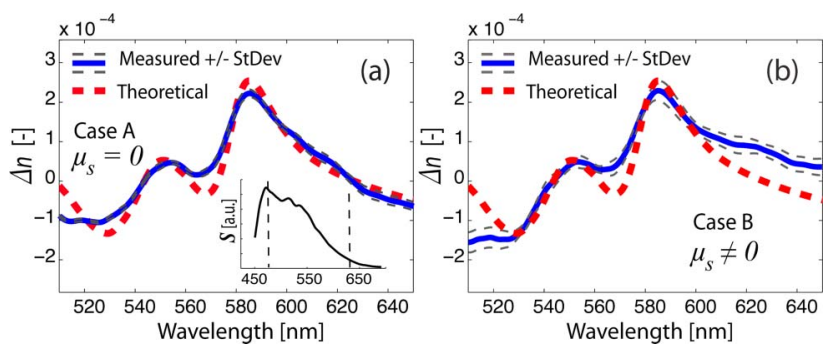

Fig. 2. (Color online) Measured change in the real part of the $\mathrm{RI}$ of the Hb phantoms (a) without scattering (case A) and (b) with scattering (case $B$ ). The theoretical change in the real part of the RI of $\mathrm{Hb}$, with a concentration of $40 \mathrm{~g} / \mathrm{L}$, is also plotted. The inset illustrates the source's spectrum, $S$, with the dotted lines denoting the bandwidth used for estimating concentration using the real part of the RI. 


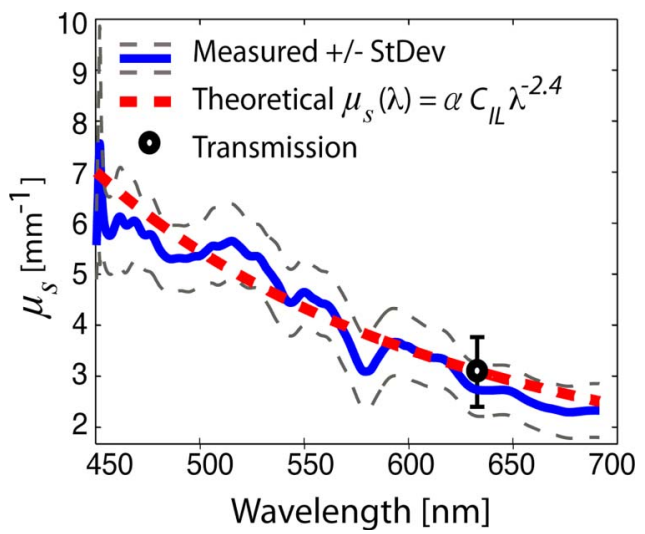

Fig. 3. (Color online) Measured and theoretical scattering coefficient of $10 \%$ IL with a $10.75 \%$ concentration.

cumulative absorption for cases $\mathrm{A}$ and $\mathrm{B}$ and the theoretical absorption from $\mathrm{Hb}$ are shown in Fig. 1(a). Figure 1(b) shows the measured total attenuation coefficients, along with the theoretical absorption coefficient of $\mathrm{Hb}$.

Next, the nonlinear phase term was analyzed to determine the real part of the RI. After dispersion effects inherent in the system and resulting from a water phantom were accounted for, the residual phase, $\Delta \phi$, from $\mathrm{Hb}$ phantoms was obtained using the method described above. Then, by using a subtractive KK relation [11], $\Delta n(\omega)$ values, which depend linearly on concentration, were determined, thus allowing assessment of $\mathrm{Hb}$ concentrations via a linear least-squares-fitting method. Here, a limited bandwidth was used to avoid regions of low intensity due to the source, as seen in the inset of Fig. 2, and due to regions of substantial $\mathrm{Hb}$ attenuation at the lower wavelengths, as seen in Fig. 1. Based on analysis of the nonlinear phase, we obtained $C=39.80 \pm$ $1.00 \mathrm{~g} / \mathrm{L}$ for case A and $C=41.09 \pm 1.93 \mathrm{~g} / \mathrm{L}$ for case $\mathrm{B}$; both are in excellent agreement with the known $\mathrm{Hb}$ concentration. The measured and theoretical $\Delta n(\omega)$ values are shown in Fig. 2(a) for case A and in Fig. 2(b) for case B. Note that the theoretical and measured real RI profiles are in good agreement for both cases; however, some discrepancies are observed at the edges of the range of analysis for case B, which need further investigation. From these results, the imaginary part of the RI and, hence, $\mu_{a}$, may be determined independently of scattering. Because the calculated concentrations for both cases are approximately equal, the absorption coefficient for case $\mathrm{B}$ (not shown) is the same as $\mu_{\mathrm{tot}}=\mu_{a}$ for case A in Fig. 1(b).

The scattering coefficient of the scattering sample (case B) can now be determined from the imaginary part of the RI. The obtained $\mu_{a}$ is subtracted from $\mu_{\text {tot }}$ (determined from the TF analysis) to give $\mu_{s}$ (Fig. 3). These results were then compared to a model of the form $\mu_{s}=\alpha C_{\mathrm{IL}} \lambda^{-2.4}$, which has been shown to be an adequate model of $10 \%$ IL scattering for a wavelength range of $400-1100 \mathrm{~nm}$ and concentrations of 4\%-17\% [12]. Here, the concentration of $10 \% \mathrm{IL}, C_{\mathrm{IL}}$, is defined as the ratio between the volume of $10 \% \mathrm{IL}$ and the total aqueous solution. Further, $\alpha$ is a concentration-independent factor, which, in part, accounts for inconsistencies in the manufacturing process and must, therefore, be experimentally measured for each batch of $10 \% \mathrm{IL}$. To obtain $\alpha$, a $\mathrm{He}-\mathrm{Ne}$ laser was used in a transmission experiment, where $10 \%$ IL was diluted to different concentrations in $5 \mathrm{~mm}$ thick glass cuvettes. The average value $\alpha=$ $9.62+/-2.8$ was used to determine a best-fit $10 \%$ IL concentration from the measured $\mu_{s}$, for case B. This analysis gave $C_{\mathrm{IL}}=10.40+/-1.40 \%$, in very good agreement with the actual $10 \%$ IL concentration $(10.75 \%)$. Note that, by using the average $\alpha$ for $C_{\mathrm{IL}}=10.75 \%$, we obtain an ideal $\mu_{s}=3.10+/-0.9 \mathrm{~mm}^{-1}$ at $\lambda=632.8 \mathrm{~nm}$ : this value is used as a calibration point to assess the theoretical $\mu_{s}$ illustrated in Fig. 3 .

In conclusion, we have derived a relation that yields the real part of the RI from the nonlinear phase term of interferometric signals in LCI. Using this relation, in combination with $\mathrm{TF}$ analysis, the absorption and scattering coefficients may be separated for samples that both scatter and absorb light, without a priori information. We validated the approach by analyzing the composition of $\mathrm{Hb}$ phantoms with and without scattering present. The results show that this method may help overcome the issues that currently prevent depth-resolved interferometric techniques from quantifying absorption in turbid samples. Further, this method may be readily extended to SOCT for imaging, in addition to allowing quantification of chromophores in blood and tissue in vivo.

This research has been supported by grants from the National Institutes of Health (NIH) (NCI 1 R01 CA138594-01).

\section{References}

1. M. Friebel, A. Roggan, G. Müller, and M. Meinke, J. Biomed. Opt. 11, 034021 (2006).

2. B. Hermann, B. Hofer, C. Meier, and W. Drexler, Opt. Express 17, 24162 (2009).

3. D. Faber, E. Mik, M. Aalders, and T. van Leeuwen, Opt. Lett. 30, 1015 (2005).

4. F. Robles, R. N. Graf, and A. Wax, Opt. Express 17, 6799 (2009).

5. D. J. Faber and T. van Leeuwen, Opt. Lett. 34, 1435 (2009).

6. Y. Park, T. Yamauchi, W. Choi, R. Dasari, and M. S. Feld, Opt. Lett. 34, 3668 (2009).

7. J. A. Izatt and M. A. Choma, in Optical Coherence Tomography: Technology and Applications, W. Drexler and J. G. Fujimoto, eds. (Springer, 2008), pp. 47-72.

8. F. Robles and A. Wax, Opt. Lett. 35, 360 (2010).

9. Y. Zhu, N. Terry, and A. Wax, Opt. Lett. 34, 3196 (2009).

10. S. Prahl, "Optical Absorption of Hemoglobin," http://omlc .ogi.edu/spectra/hemoglobin/.

11. D. Faber, M. Aalders, E. Mik, B. Hooper, M. van Gemert, and T. van Leeuwen, Phys. Rev. Lett. 93, 028102 (2004).

12. H. J. van Staveren, J. M. Moes, J. van Marle, and S. A. Prahl, Appl. Opt. 30, 4507 (1991). 